\title{
R-CHOP Plus Methotrexate Regimen
}

National Cancer Institute

\section{Source}

National Cancer Institute. R-CHOP Plus Methotrexate Regimen. NCI Thesaurus. Code C156246.

An immunochemotherapy regimen consisting of rituximab, cyclophosphamide, hydroxydaunorubicin hydrochloride (doxorubicin hydrochloride), vincristine (Oncovin), prednisone (R-CHOP), and methotrexate used to treat certain types of lymphomas. 